\title{
STUDY ON FUNCTIONING OF EFFERENT AUDITORY PATHWAY IN MENTALLY CHALLENGED.
}

\section{K Yerraguntla \\ VJ Vanila \\ R Bellur}

\section{Correspondence to}

Dr. Krishna Y

Assoc. Professor

Dept. of Speech and Hearing

MCOAHS, MAHE

Manipal, Karnataka, India

E-mail: krishna.y@maniapl.edu

\begin{abstract}
Objective:
The main objective of the study is to compare the amount of suppression of TEOAE's with contralateral stimulation in mentally challenged children and age matched normal children and further to study the functioning of the efferent auditory pathway in children with delayed maturation of the auditory pathway especially in individuals with non-Down's syndrome..
\end{abstract}

Material and methods:

The present study was aimed at investigating the contralateral suppression of Transient Evoked Otoacoustic Emissions (TEOAEs) in normal children as compared with that of developmentally delayed. 15 mentally challenged children in the age range of 7-12 years and gender matched normal children participated in the study.

Results:

Results suggest reduced contralateral suppression of TEOAEs in mentally challenged children as compared to their normal counterparts. Contralateral suppression was observed at more in left ear compared to right ear. The mid frequencies were observed to have greater suppression in both ears.

Conclusion:

The results of the current study reinforces the contention that contralateral suppression of TEOAEs is reduced in children with delayed maturation and provides insight for confirming the risk of auditory processing difficulties and speech perception in noise. The association between contralateral acoustic stimulation and OAE allows easy, non-invasive study of auditory efferent mechanisms and seems to be clinically feasible for evaluating cochlear status and auditory efferent function.

Ke y word s: efferent auditory pathway, mentally challenged, otoacoustic emissions

\section{INTRODUCTION:}

Hearing is one of the most important sensory functions of the body which enables individuals to have an effective communication. It has been demonstrated that the ear, besides receiving sounds, also generates sounds. These sounds emitted by the ear are called as OtoAcoustic Emissions (OAEs). These emissions were first described by Kemp in 1978.1 Human auditory system has both afferent and efferent pathways. The efferent auditory system is a descending bundle, which originates from the auditory cortex and terminates at the sensory cells of the organ of Corti. In 1946, Rasmussen reported the discovery of the Olivo-Cochlear (OC) system.2 It was reported that the outer hair cell $(\mathrm{OHC})$ innervations is primarily from the Medial Olivo-Cochlear efferent System (MOCS). Since OAEs are thought to reflect these dynamic properties, it has been hypothesized that activating the medial efferent system would produce alterations to cochlear micromechanics and hence, to OAEs. The first description of the active mechanical behavior of outer hair cells was made over 20 years ago. ${ }^{3}$ In the literature, it is reported that afferent auditory fibers from the cochlear nuclei project mainly to the contralateral MSO nuclei. 4 It is well established that the amplitude of both types of Evoked Otoacoustic Emissions (TEOAEs as well as the DPOAEs) can be suppressed when simultaneous contralateral sound stimulation is applied 5,6 and is due to mediation of medial efferent system.

Effect of age and maturation of the medial efferent system on the amplitude of TEOAE was studied and reported that amplitude increased with the age and maturation.7,8 Since, fibers of MOCS predominantly innervate $\mathrm{OHC}^{\prime} \mathrm{s}$, it is presumed that they exert their suppressive influence via this pathway and most probably by interfering with cochlear amplifier function. 9 Hence, from the literature, it is observed that functioning of MOCS is best studied in individuals with Downs syndrome or in individual with Mental Retardation 10 or delayed maturation.

In the literature, TEOAE studies done on Down's syndrome and mentally retarded individuals showed reduced emissions.11-17 Hood et al study observed reduced TEOAEs in individuals with auditory neuropathy and commented that the poor efferent responses observed could be due to compromised afferent input to the OCR pathway. 18 They also commented that, efferent suppression of otoacoustic emissions could be used as a differential measure of auditory function in patients with auditory neuropathy/dys-synchrony. The current study was aimed to explore TEOAE suppression with contralateral stimulation in mentally challenged children compared with age and gender matched group of normal children.

\section{MATERIAL AND METHODS:}

Fifteen children with normal hearing sensitivity and mentally challenged comprised of experimental group. Their age ranged from 7-12 years and their IQ from 35-50 as certified by a qualified Clinical Psychologist. Children with middle ear disorders were excluded from the study. Children with normal hearing and with the presence of TEOAE in both ears were included in the study. Age and gender matched normal children formed the control group who were evaluated to rule out any hearing loss and or middle ear disorders.

A calibrated double channel diagnostic audiometer, immitance audiometer and Biologic Scout report program (V 3.02) systems were used. Prior to the instrumental evaluation, a visual examination of the ear canal of both ears was done using an otoscope to rule out any outer ear abnormalities. Pure tone thresholds were obtained at octave frequencies using Modified Hughson - Westlake procedure19 in a sound treated room. Tympanometry and reflexometry were carried out with a probe tone of $226 \mathrm{~Hz}$. A good probe fit was ensured while testing for OAEs and data that had 50\% above reproducibility, $90 \%$ stability, $+3 \mathrm{~dB}$ S/N ratio, artifact rejection threshold of $50 \mathrm{mPa}$ were considered.

After clear instructions and verbal consent from the participant, an appropriate probe fit was obtained by observing the stimulus spectrum. Initially, TEOAEs were recorded by eliciting them with $80 \mathrm{sec}$ linear clicks at 60-65 dBSPL. For contralateral stimulation, white noise was delivered through headphones from an audiometer. The continuous noise at $5 \mathrm{~dB}$ above click stimulus was presented in the contralateral ear simultaneously. An average of 200 responses repeated twice was considered for each recording. Average TEOAE waveforms were obtained to measure the reproducibility, with reproducibility greater than $50 \%$ being considered. Data for frequencies $1000 \mathrm{~Hz}$, $1500 \mathrm{~Hz}, 2000 \mathrm{~Hz}, 3000 \mathrm{~Hz}$ and $4000 \mathrm{~Hz}$ were recorded. Suppression of TEOAEs was explored by subtracting the 'with noise' average from 'without noise average'. Data was subjected to statistical analysis using Students t- test and Mann-Whitney-U test to compare the TEOAE suppression results of normal hearing children with that of mentally challenged children. 
RESULTS:

To study the functioning of the efferent auditory pathway in individuals with mentally challenged, 15 participants with mentally challenged and age matched normals were studied using Transient Otoacoustic Emissions and its suppression. The Mean, Standard Deviation, $p$ values and $z$ values of contralateral suppression measures in right ear between normal \& mentally challenged children for frequencies $500 \mathrm{~Hz}, 1 \mathrm{kHz}$ $1.5 \mathrm{kHz}, 2 \mathrm{kHz}, 3 \mathrm{kHz}$ and $4 \mathrm{kHz}$ are given in Table 1 and Fig- 1 .

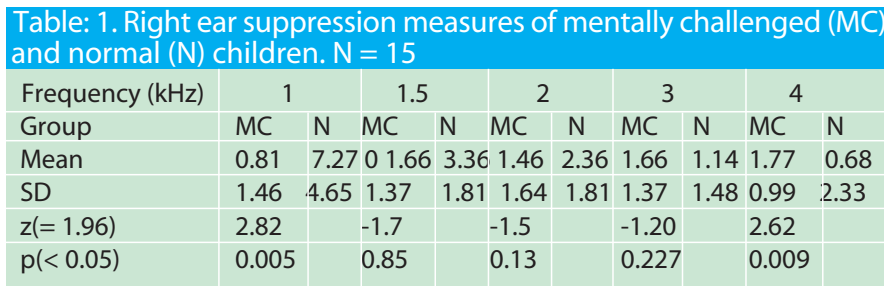

Fig: 1. Mean values of TEOAE suppression between mentally challenged and normal children in right ear.

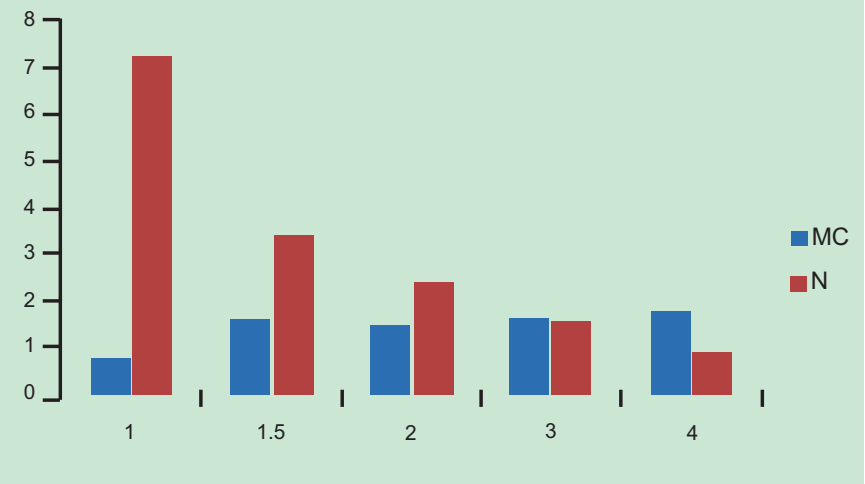

From Table- 1, it is observed that, in right ear at $1 \mathrm{kHz}$ the mean contralateral suppression is of statistically significant difference $(p<$ 0.05 ) between the two groups (normal hearing individuals and mentally challenged), confirming the presence of greater suppression in normal children than in mentally challenged children. At 1.5 and $2 \mathrm{kHz}$, greater suppression in normal individuals is observed as compared to mentally challenged children; however, it is not statistically significant. At 3 $\mathrm{kHz}$ and $4 \mathrm{kHz}$, it is interesting to note that greater suppression is seen in mentally challenged children as compared to the normal group however, the difference is not statistically significant. On scrutiny of the results from Table- 2 , it is can be observed that, in left ear at 1, 2 and $3 \mathrm{kHz}$, normals had greater suppression compared

\begin{tabular}{|c|c|c|c|c|c|c|c|c|c|c|}
\hline Frequency (kHz) & 1 & & 1.5 & & 2 & 2 & 3 & & 4 & \\
\hline Group & MC & $\mathrm{N}$ & MC & $\mathrm{N}$ & MC & $\mathrm{N}$ & MC & $\mathrm{N}$ & MC & $\mathrm{N}$ \\
\hline Mean & 1.33 & 2.50 & 1.13 & 2.70 & 0.11 & 1.55 & 1.67 & 0.17 & 2.17 & 2.50 \\
\hline SD & 1.93 & 1.40 & 2.38 & 2.16 & 52.05 & 2.00 & 1.36 & 1.71 & 1.00 & 1.70 \\
\hline$z(=1.96)$ & -2.07 & & -1.598 & & -2.26 & & -2.34 & & -0.104 & \\
\hline $\mathrm{p}(<0.05)$ & 0.038 & & 0.110 & & 0.024 & & 0.019 & & 0.917 & \\
\hline
\end{tabular}

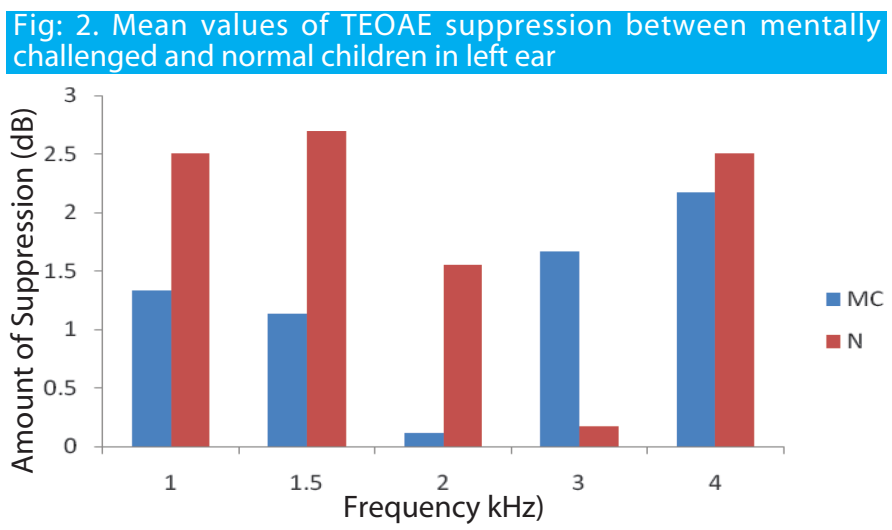

to mentally challenged individuals and the difference being statistically significant. However, at 1.5, and $4 \mathrm{kHz}$, although normals had greater suppression, the difference was not statistically significant. From the data, it is interpreted that, majority of the frequencies show greater suppression in normal than in mentally challenged children.

DISCUSSION:

The current study was done to compare the amount of suppression of TEOAE's with contralateral stimulation in normal children and mentally challenged children of the age matched group to study the functioning of the efferent auditory pathway. From the results it is observed that, the contralateral suppression was more in majority of the frequencies in normal children and reduced in mentally challenged children, implies that in the mentally challenged children, there is a delay in maturation of the efferent Medial Olivo-Cochlear Bundle (MOCB).

Significant suppression effect demonstrated in the present study is in agreement with the reports of Andersson et al.15 From studies, it is evident that normal and mature MCOB and efferent auditory pathway are essential to elicit OAE suppression. 3,4,9,19 The maturation delays observed in Down's syndrome and mentally challenged do affect the efferent auditory pathway.

Studies on individuals with Down's syndrome have commented that delayed maturation of efferent auditory pathway could be due to reduction in brain myelination beyond early childhood in developmentally delayed children. 11-17,20 Further, Down's syndrome children have different degrees of developmental disabilities, developmental delays and developmental brain abnormalities with CNS maturation delay and cortical digenesis. 20 From the results of developmental, neuro imaging studies, it can be inferred that poor efferent responses are related to the compromised afferent input to the olivocochlear bundle pathway in mentally challenged children and support the hypothesis that "poor efferent responses are related to compromised afferent input to the olivocochlear bundle" as quoted in the literature. 18 The compromised efferent auditory pathway thus plays a role in TEOAE suppression and leading to reduced TEOAE suppression when contralateral stimulus is given. As observed in the current study, reduced TEOAE suppression in most of the frequencies could be due to maturational abnormalities.

In the literature, studies have concentrated more in the Down's syndrome category of mentally challenged group and have reported of reduced suppression. The present study, excluded the Down's syndrome subjects and considered only the mentally challenged to see if similar results are observed as in Down's group. Results do confirm similar suppression pattern (reduced) in the mentally challenged group exclusive of Down's syndrome and enable us to state that the contralateral suppression is lesser in all categories of mentally challenged and attributable to lack of maturation of efferent auditory pathway or abnormalities in MOCB pathway as reported in the literature. The results of the current study also endorse the use of efferent suppression of otoacoustic emissions as a differential measure of auditory function in patients with developmental delay.

\section{CONCLUSION:}

In conclusion, there exists evidence that the assessment of the medial olivocochlear system, by recording OAEs under contralateral acoustic stimulation helps in the diagnosis of auditory maturation delays, especially efferent audiotry pathway, auditory processing disorders and other hearing disorders related to delayed maturation of the efferent auditory pathway. It also acts as a non-invasive tool for hearing screening of infants who are at risk for hearing loss and also at risk for hearing problems due to neural maturation.

REFERENCES:

1. Kemp DT. Stimulated acoustic emissions from within the human auditory system. J Acoust Soc Am 1978; 64: 1386-91.

2. Rasmussen GL. The olivary peduncle and other fiber projections of the superior olivary complex. J Comp Neurol 1946; 84: 141-219.

3. Brownell WE. Outer hair cell electromotility and otoacoustic emissions. Ear Hear 1990; 11: 82-92.

4. Sahley T, Nodar R, Musiek F. Neuroanatomy of the auditory pathways. Efferent Auditory System: Structure and Function. London: Singular Publishing Group, Inc 1997. 
5. Ryan S, Kemp DT, Hinchcliffe R. The influence of contralateral acoustic stimulation on click-evoked otoacoustic emissions in humans. Br J Audiol 1991; 25: 391-7.

6. Williams EA, Brookes GB, Prasher DK. Effects of olivocochlear bundle section on otoacoustic emissions in humans: efferent effects in comparison with control subjects. Acta Otolaryngol 1994; 114: 121-9.

7. Morlet T, Goforth L, Hood LJ, Ferber C, Duclaux R, Berlin Cl. Development of human cochlear active mechanism asymmetry: involvement of the medial olivocochlear system? Hear Res 1999; 137: 179.

8. Morlet T, Collet L, Salle B, Morgon A. Functional maturation of cochlear active mechanisms and of the medial olivocochlear system in humans. Acta Otolaryngol 1993; 113: 271-7.

9. Keppler H, Dhooge I, Corthals P, Maes L, D'haenens W, Bockstael $A$, et al. The effects of aging on evoked otoacoustic emissions and efferent suppression of transient evoked otoacoustic emissions. Clin Neurophysiol 2010; 121: 359-65.

10. AAMR. Mental Retardation: Definition, Classification, and Systems of Supports. (10th ed). Washington, DC: American Association on Mental Retardation 2002.

11. Pujol J, López-Sala A, Sebastián-Gallés N, Deus J, Cardoner N, Soriano-Mas C, et al. Delayed myelination in children with developmental delay detected by volumetric MRI Neuroimage 2004; 22: 897-903.

12. Wisniewski KE, Schmidt-Sidor B. Postnatal delay of myelin formation in brains from Down syndrome infants and children. Clin
Neuropathol 1989; 8: 55-62.

13. Koo BK, Blaser S, Harwood-Nash D, Becker LE, Murphy EG. Magnetic resonance imaging evaluation of delayed myelination in down syndrome: a case report and review of the literature. J Child Neurol 1992; 7: 417-21.

14. Driscoll C, Kei J, Bates D, McPherson B. Tympanometry and TEOAE testing of children with down syndrome in special Schools. Aust NZ J Audiol 2003; 25: 85-93.

15. Andersson E, Arlinger S, Jacobsson S. Evaluation of OAE-recording as a complementary test method for adults with moderate to profound mental retardation. Scand Audiol 2000; 29: 120-6.

16. Maroudias N, Economides J, Christodoulou P, Helidonis E. A study on the otoscopical and audiological findings in patients with down's syndrome in Greece. Int J Pediatr Otorhinolaryngol 1994; 29: 43-9.

17. Squires N, Ollo C, Jordan R. Auditory brain stem responses in the mentally retarded: audiometric correlates. Ear Hear 1986; 7: 8392.

18. Hood LJ, Berlin Cl, Bordelon J, Rose K. Patients with auditory neuropathy/dys-synchrony lack efferent suppression of transient evoked otoacoustic emissions. J Am Acad Audiol 2003; 14: $302-$ 13.

19. Dewson JH. Efferent olivocochlear sone relationships to stimulus discrimination in noise. J Neurophysiol 1968; 31: 122-30.

20 Wisniewski K. Down syndrome children often have brain with maturation delay, retardation of growth, and cortical dysgenesis. Am J Med Genet Suppl 1990; 7: 274-81. 